\title{
Advances in nuclear reaction calculations by incorporating information from nuclear mean-field theories
}

\author{
Toshihiko Kawano ${ }^{\mathrm{a}}$ \\ Theoretical Division, Los Alamos National Laboratory, Los Alamos, New Mexico 87545, USA
}

\begin{abstract}
Mean-field model calculations for nuclear structure theories are combined with the statistical Hauser-Feshbach code in order to improve predictive capabilities of nuclear reaction for experimentally unknown cross sections. Utilizing the mean-field calculation results we calculate second moments of matrix elements for the residual interaction. The second moments are applied to a microscopic level density model based on the random matrix theory. An example is shown for the ${ }^{208} \mathrm{~Pb}$ level density calculation.
\end{abstract}

\section{Introduction}

Calculations of the statistical Hauser-Feshbach theory with the direct and pre-equilibrium processes require many model inputs such as the optical potentials for particle transmission coefficients, the level densities in a continuum state, the photon strength functions for $\gamma$-ray transmission coefficients, the fission barriers for the fission decay channel, and so on. We often employ model parameters that are estimated phenomenologically, or sometimes we derive them semi-microscopically with nuclear structure theories and provide the results in a tabulated form. Although pre-calculated model inputs are available in a computer readable format [1], we still envision that combining nuclear structure models with the nuclear reaction calculations will be very attractive for further development of nuclear reaction codes. An example is the mean-field model calculation for the neutron direct/semi-direct (DSD) capture model developed at Los Alamos [2], where the Hartree-Fock (HF) BCS theory is used for calculating bound-neutron wave-functions as well as the particle occupation probabilities in a deformed nucleus. Recently this technique was extended to the Finite-Range Droplet Model (FRDM) [3] and the model was unified with the statistical Hauser-Feshbach code $\mathrm{CoH}_{3}$ at Los Alamos National Laboratory (LANL) [4].

Since modern fully-microscopic nuclear structure calculations often require large-scale computations, it might still be a long way until we combine nuclear structure and reaction calculations in a common framework in practical applications. For example, recent studies on a fully microscopic description of the particle scattering process are promising to reduce phenomenological inputs $[5,6]$. Nevertheless, we limit ourselves to semi-microscopic approaches so that we are able to perform calculations of the nuclear structure and reaction models simultaneously. By solving the Schrödinger equation for a one-body potential, we have the single particle wave-functions $u(r)$, which can be inserted into the DWBA matrix elements $\left\langle u(r)|V| \chi^{(+)}(r)\right\rangle$ in the direct reaction. The single-particle

a e-mail: kawano@lanl.gov wave-functions are the chief ingredient of matrix elements for the residual interaction (e.g. see Eq. (8.1) of [7])

$$
H^{\prime}=\frac{1}{4} \sum\langle\alpha \beta|\mathcal{V}| \gamma \delta\rangle c_{\alpha}^{\dagger} c_{\beta}^{\dagger} c_{\delta} c_{\gamma}
$$

which plays an important role when the complexity of the system is evolved, such as in the quantum mechanical preequilibrium theories [8].

In this paper we discuss possible connections between the nuclear reaction and structure models, with particular focus on a nuclear reaction model code development. Ullmann et al. [9] showed an enhancement of neutron radiative capture cross sections for deformed nuclei due to the M1 scissors mode. This striking observation indicates that more realistic spin and parity distributions in the continuum is crucial, because the neutron captured state decays to the final state with different parity depending on the multipolarity of the $\gamma$-ray strength functions. We calculate the microscopic nuclear level densities that include the residual interaction of Eq. (1) based on the random matrix theory [10-12]. This microscopic level density model was originally developed for calculating partial level densities with a fixed number of excitons in the quantum mechanical multistep direct reactions. By applying the same technique to the total level density, the modelling of the compound and pre-compound processes can be more consistent.

\section{LANL Hauser-Feshbach Code, $\mathrm{CoH}_{3}$}

The coupled-channels Hauser-Feshbach theory code, $\mathrm{CoH}_{3}$ [4], calculates the optical model and the compound nuclear reactions in the $\mathrm{keV}$ to $\mathrm{MeV}$ energy range. $\mathrm{CoH}_{3}$ is extensively utilized in the nuclear data evaluation above the resonance region at LANL, as well as for the interpretation of experimental data at LANSCE (Los Alamos Neutron Science Center), e.g., the neutron capture measurements with the DANCE detector $[9,13]$, and the $\gamma$-ray production measurements with the GEANIE detector $[14,15]$.

The optical model part includes the coupled-channels theory for the rotational and vibrational inelastic excitation

(C) The Authors, published by EDP Sciences. This is an Open Access article distributed under the terms of the Creative Commons Attribution License 4.0 (http://creativecommons.org/licenses/by/4.0/). 


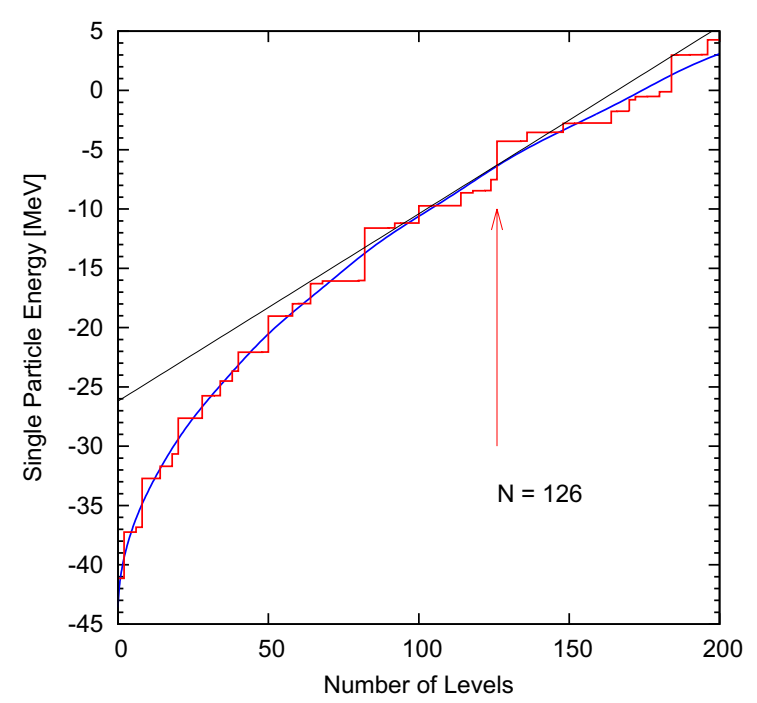

Figure 1. The single-particle energies of ${ }^{208} \mathrm{~Pb}$ calculated with the FRDM folding Yukawa potential. The red staircase plot shows the actual single-particle energies, and blue curve is the smoothed one with Strutinsky's method.

[16]. In the compound reaction calculation, it adopts the width fluctuation correction model by Moldauer [17] with the updated parameterization based on the Gaussian Orthogonal Ensemble (GOE) [18,19]. When applied to a deformed nucleus, the coupled-channels scattering matrix is diagonalized and the width fluctuation calculation is performed in the diagonalized space [20], the so-called Engelbrecht-Weidenmüller transformation [21]. The code runs in both a regular deterministic mode and a Monte Carlo mode in which particles and $\gamma$-rays are generated on an event-by-event basis [22].

The code includes two mean-field theories; FRDM [3] and the Skyrme HF-BCS [23]. In $\mathrm{CoH}_{3}$, the singleparticle potential $V=V_{1}+V_{\text {s.o }}+V_{\mathrm{C}}$ of FRDM, where the central part $V_{1}$ is calculated in terms of the folded Yukawa potential

$$
V_{1}(\mathbf{r})=-\frac{V_{0}}{4 \pi a^{3}} \int \frac{\exp \left(-\left|\mathbf{r}-\mathbf{r}^{\prime}\right| / a\right)}{\left|\mathbf{r}-\mathbf{r}^{\prime}\right| / a} d^{3} r^{\prime}
$$

can be used as an initial potential in the Hartree-Fock iteration. The integration of Eq. (2) is performed over the volume for a given shape, $V_{0}$ is the potential depth, and $a$ is the range of the Yukawa interaction. The single particle wave-functions are given directly from the folded Yukawa potential in the FRDM case, or after the Hartree-Fock iteration starting with Eq. (2).

So far there are two models in $\mathrm{CoH}_{3}$ that utilize the mean-field calculation outputs. As mentioned before, the single-particle wave-functions of FRDM and HF-BCS can be used in the DSD model [2]. In addition, the singleparticle state density parameter $g$ in the pre-equilibrium model can be estimated by Strutinsky's method in FRDM, as it is given by smoothed variation in the single-particle energies [24]. In Fig. 1 the neutron single-particle energies calculated with FRDM for ${ }^{208} \mathrm{~Pb}$ are shown by the staircase plot, and the $g$ parameter is calculated as a tangent at the Fermi energy. For the ${ }^{208} \mathrm{~Pb}$ case, $g=6.3 \mathrm{MeV}^{-1}$ for the neutron shell. With this technique we are able to eliminate a very crude estimation of the $g$ parameter, such as $g_{(N, Z)}=(N, Z) / 15 \mathrm{MeV}^{-1}$, where $Z$ or $N$ is the nucleon number. This simple relation gives the overestimated value of $126 / 15=8.4 \mathrm{MeV}^{-1}$.

\section{Level density based on random matrix theory}

\subsection{Unperturbed level density}

The single-particle spectrum for the one-body potential provides a basis of the microscopic level density calculation by counting the number of particle-hole configurations. The calculation of combinatorial level density is very fast unless the total number of particles is large. However, such calculation does not include particle-hole interactions. An approximation to the shell model level density based on the random matrix theory was proposed by Pluhař and Weidenmüller [10,25], then applied to practical calculations $[11,12]$. In this technique the Hamiltonian for the nuclear system has the singleparticle part $H_{0}$ and the residual interaction $V$,

$$
H=H_{0}+V \text {. }
$$

The eigenfunction and eigenvalue of the single-particle Hamiltonian satisfy

$$
\left(H_{0}-\epsilon_{m \mu}\right)|m \mu\rangle=0,
$$

where $m$ is the total particle and hole numbers $(m=2$ for the $1 \mathrm{p}-1 \mathrm{~h}$ configuration, 4 for $2 \mathrm{p}-2 \mathrm{~h}$, etc.), and $\mu$ is the other quantum numbers. The unperturbed state density $\rho_{m}^{(0)}(E, M)$ in the $M$-scheme is given by

$$
\rho_{m}^{(0)}(E, M)=\sum_{\mu} \delta\left(E-\epsilon_{m \mu}\right)
$$

where $M$ is the sum of the $z$-component of the total angular momentum of each single-particle state. We drop a trivial index for parity $\Pi$ unless given explicitly otherwise. The $J$-scheme level density is given by the well-known relation

$$
\rho_{m}^{(0)}(E, J)=\rho_{m}^{(0)}(E, M=J)-\rho_{m}^{(0)}(E, M=J+1),
$$

and the observed total level density is

$$
\rho^{(0)}(E)=\sum_{m J \Pi} \rho_{m}^{(0)}(E, J) .
$$

The calculated unperturbed level density $\rho^{(0)}(E)$ for ${ }^{208} \mathrm{~Pb}$ is shown in Fig. 2 by the blue histogram. The single particle eigenvalues are for the FRDM folding Yukawa potential, and the particle-hole configuration energies are $200-\mathrm{keV}$ binned. The microscopic level density is compared with a phenomenological model of level density based on the Gilbert-Cameron formula [26], shown by the solid line. The parity unevenness, $\rho^{+}(E) /\left[\rho^{+}(E)+\right.$ $\left.\rho^{-}(E)\right]$, is shown in Fig. 3 .

\subsection{Second moments of matrix elements for residual interaction}

The matrix elements of the residual interactions $V$ are assumed to form a Gaussian Orthogonal ensemble (GOE) characterized by the zero ensemble average

$$
\overline{V_{m \mu, n v}}=0,
$$




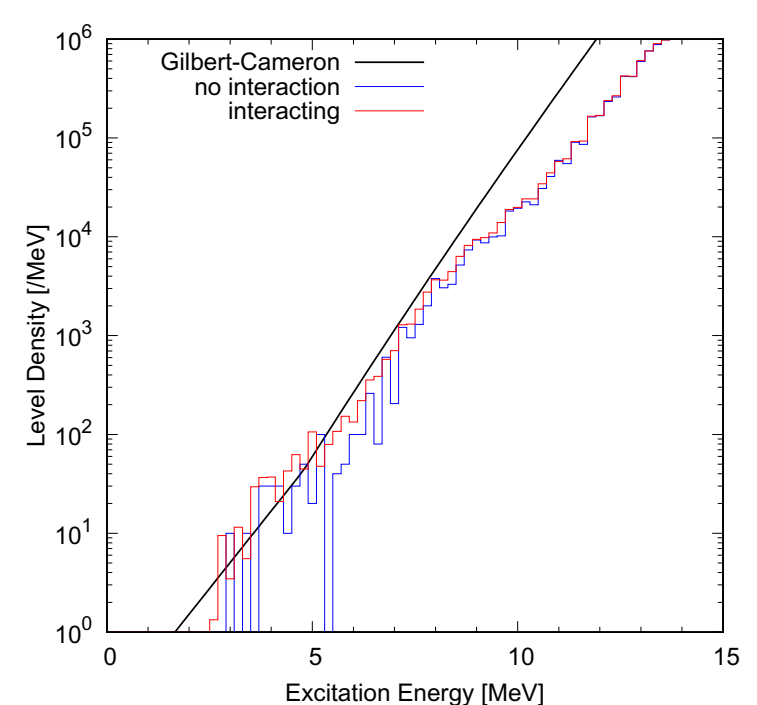

Figure 2. The calculated level density of ${ }^{208} \mathrm{~Pb}$ using the FRDM folding Yukawa potential. The blue histogram is the unperturbed level density $\rho^{(0)}(E)$, and the red histogram shows the case when the $\delta$-interaction is introduced.

and the second moment

$$
\begin{aligned}
& \overline{V_{m \mu, n v} V_{m^{\prime} \mu^{\prime}, n^{\prime} v^{\prime}}} \\
= & \mathcal{M}_{m n}\left(\delta_{m m^{\prime}} \delta_{n n^{\prime}} \delta_{\mu \mu^{\prime}} \delta_{v v^{\prime}}+\delta_{m n^{\prime}} \delta_{n m^{\prime}} \delta_{\mu v^{\prime}} \delta_{v \mu^{\prime}}\right) .
\end{aligned}
$$

Because our potential is axially deformed, and eigenfunctions are expanded in the cylindrical harmonic oscillator basis, all single-particle states are labeled by the $z$ component of the total angular momentum, $\Omega$. The second moment $\mathcal{M}_{m n}$ is first calculated by summing the matrix elements of residual interaction in the $M$-scheme. The $J$ scheme second moments are given by [12]

$$
\begin{aligned}
& \sum_{\mu \nu}\left(V_{m \mu, n v}\right)_{M}^{2}-\sum_{\mu \nu}\left(V_{m \mu, n \nu}\right)_{M+1}^{2} \\
= & \sum_{\mu \nu}\left(V_{m \mu, n \nu}\right)_{M=J}^{2} \\
= & \mathcal{M}_{m n}(J) N_{m}(J) N_{n}(J),
\end{aligned}
$$

where

$$
N_{m}(J)=\int \rho_{m}^{(0)}(E, J) d E
$$

To calculate the ensemble average of the second moments for the residual interaction, we first assume the $\delta$-interaction for simplicity

$$
\langle\alpha \beta|\mathcal{V}| \gamma \delta\rangle=\frac{V_{0}}{4 \pi} \int \phi_{\alpha}(r) \phi_{\beta}(r) \phi_{\gamma}(r) \phi_{\delta}(r) \frac{1}{r^{2}} d r
$$

where, although the notation is for the spherical case, the single-particle wave-functions are always in the axially deformed potential. We consider three cases of particle/hole interaction within the same class of $m$; the particle-particle scattering, the particle-hole scattering, and the hole-hole scattering. When a particle or a hole creates a new particle-hole pair, the class of the final state is $n=m+2$. These processes are illustrated in Fig. 4 .

These interactions account for the active part only in a many-particle many-hole configuration. To calculate the

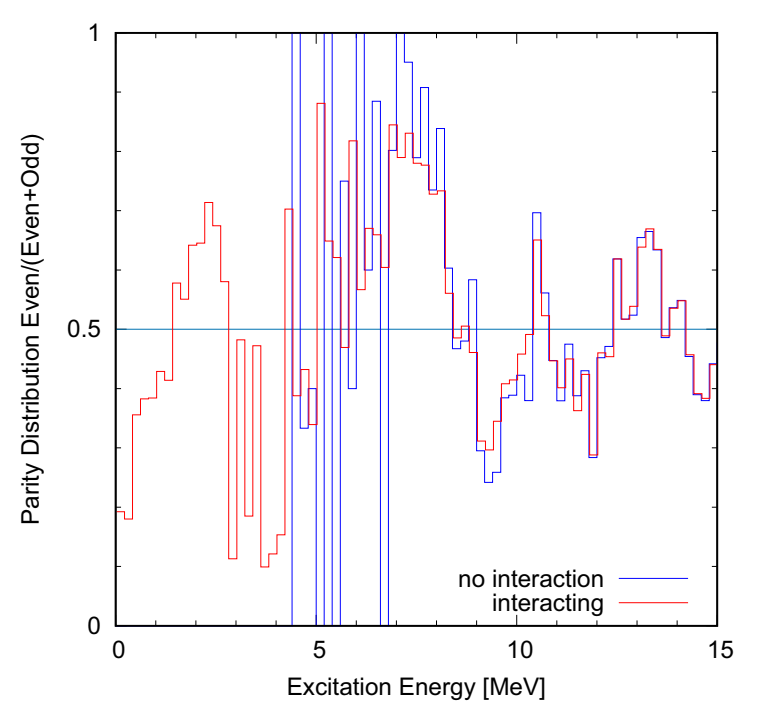

Figure 3. The calculated parity distribution for ${ }^{208} \mathrm{~Pb}$. The blue histogram is for the unperturbed level density case, and the red histogram shows the $\delta$-interaction case.

(a) scattering

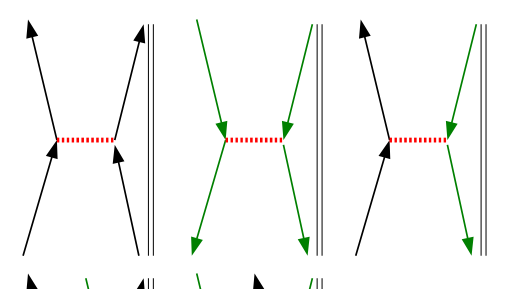

(b) pair creation

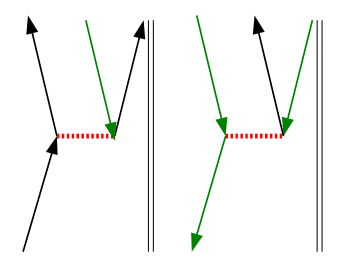

Figure 4. Interactions involved in calculating the second moments; (a) the matrix elements for particle/hole collisions, and (b) the particle-hole pair creation processes. The upward arrows are the particle, and the downward arrows are the hole. The horizontal bar indicates the interaction, and the vertical bars are the spectators.

second moments for each class of $m$, a convolution is taken over the rest of the spectators $N_{s}$. In the scattering case, it reads

$$
S_{m}(J)=\left\{\sum|\langle\alpha \beta|\mathcal{V}| \gamma \delta\rangle|^{2} \otimes N_{s}\right\}_{J},
$$

and in the pair creation case,

$$
S_{m+2}(J)=\left\{\sum|\langle\alpha|V| \beta \gamma \delta\rangle|^{2} \otimes N_{s}\right\}_{J} .
$$

The second moments are calculated by

$$
\begin{aligned}
\mathcal{M}_{m, m}(J) & =\frac{S_{m}(J)}{N_{m}(J)^{2}} \\
\mathcal{M}_{m, m+2}(J) & =\frac{S_{m+2}(J)}{N_{m}(J) N_{m+2}(J)} .
\end{aligned}
$$

The calculated second moments for ${ }^{208} \mathrm{~Pb}$, where the results are normalized by the $\delta$-interaction strength $V_{0}$, are shown in Fig. 5. $\mathcal{M}_{m, m}$ is for the scattering process, 


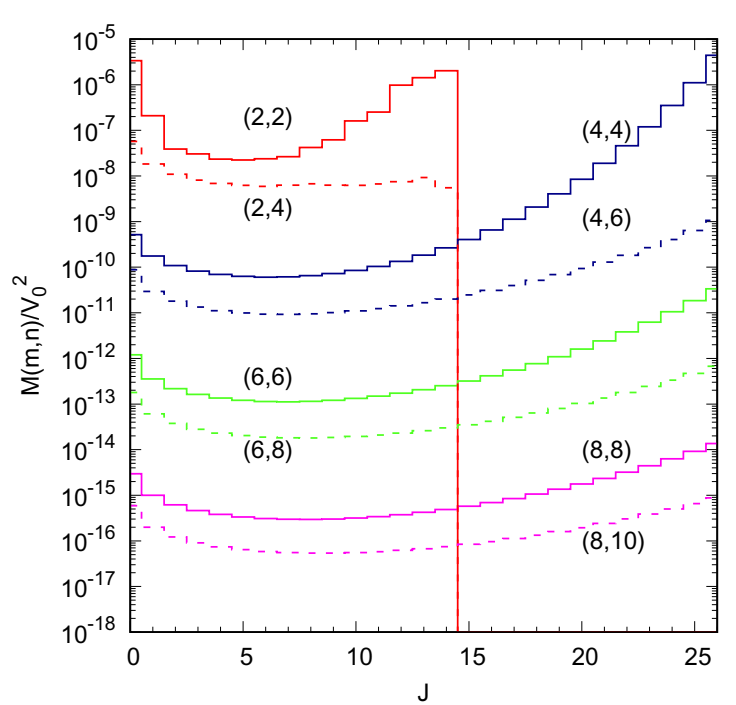

Figure 5. The calculated second moments $\mathcal{M}_{m, m}$ and $\mathcal{M}_{m, m+2}$ with the FRDM wave-functions for ${ }^{208} \mathrm{~Pb}$ as a function of $J$. Both parity states are summed.

and $\mathcal{M}_{m, m+2}$ is for the particle-hole creation process. Although this is basically repeating the calculations of Sato, Takahashi, and Yoshida [11], the current calculation has a much larger model space, and the wave-functions are taken from FRDM, instead of the spherical Woods-Saxon potential. An extension to the deformed nucleus case is straightforward.

\subsection{Saddle-point equation}

The ensemble average of level density can be evaluated by using the generating function and the Grassmann integral method [10], and the result is expressed in terms of the saddle-point value $\sigma_{m}(E, J)$,

$$
\begin{aligned}
\rho_{m}(E, J) & =-\sum_{\mu} \frac{1}{\pi} \Im \frac{1}{E-\epsilon_{m \mu}-\sigma_{m}(E, J)} \\
& =-\int \frac{1}{\pi} \Im \frac{\rho^{(0)}(E, J)}{E-\epsilon-\sigma_{m}(E, J)} d \epsilon,
\end{aligned}
$$

where $\sigma_{m}(E, J)$ is given by solving the saddle-point equation

$$
\sigma_{m}(E, J)=\sum_{n} \mathcal{M}_{m n} \int \rho_{n}^{(0)}(\epsilon, J) \frac{1}{E-\epsilon-\sigma_{n}(E, J)} d \epsilon
$$

The saddle-point equation of Eq. (18) is solved by an iterative procedure. The calculated $\sigma_{m}(E, J)$ values for $j \leq 5$ are averaged and shown in Fig. 6. The interaction is the $\delta$-interaction with $V_{0}=60 \mathrm{MeV}$. With the combinatorial calculation, the level density for a particlehole state $\mu$ is just a $\delta$-function at the energy $\epsilon_{\mu}$. While Eq. (17) implies that it is shifted by $\Re \sigma_{m}(E, J)$ and has the width of $\Im \sigma_{m}(E, J)$. Because the real part of $\sigma_{2}$ and $\sigma_{4}$ are negative below $10 \mathrm{MeV}$, as seen in Fig. 6, the $1 \mathrm{p}-1 \mathrm{~h}$ and $2 \mathrm{p}-2 \mathrm{~h}$ configurations at low excitation energies are shifted to the lower side, hence the level densities at low excitation energies are enhanced. This is shown in Fig. 2, where the observed total level density $\rho(E)=\sum_{m J \Pi} \rho_{m}(E, J)$ is plotted. In Fig. 3 one can see a smoothing effect in the parity distribution.

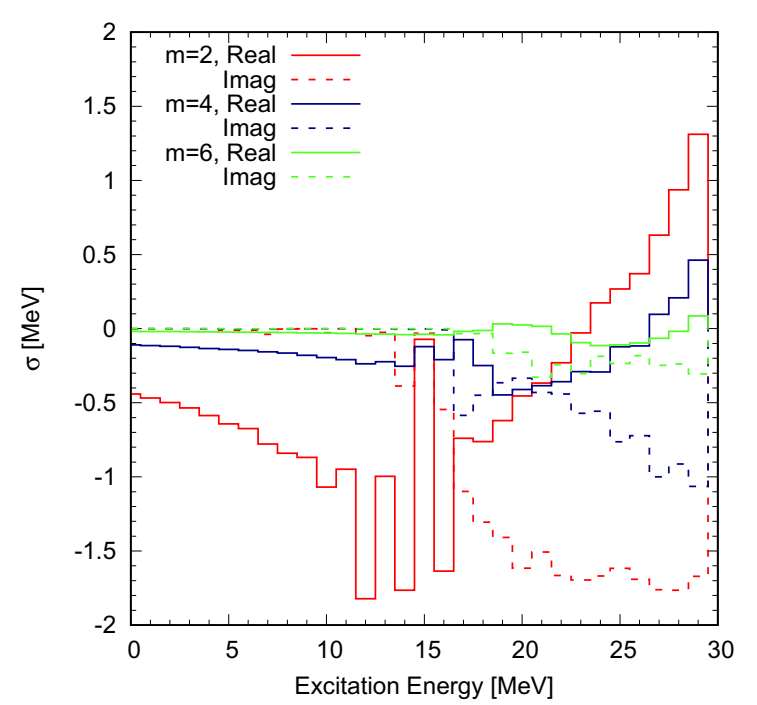

Figure 6. The averaged saddle-point value $\sigma_{m}(E, J)$ for $J \leq 5$. The solid lines are for the real part, and the dashed lines are for the imaginary part. Both parity states are summed.

The change in the level density depends on the assumed interaction. Obviously the $\delta$-interaction employed in this study is too simplistic, and should eventually be replaced by a realistic effective interaction such as the Skyrme-force to improve the level density calculation.

\section{Discussions}

Since our purpose is to combine the nuclear meanfield models with reaction calculations, it is important to minimize the computational time incurred by extra calculations. The most time consuming part of the level density calculation is the second moments. Albeit it is much faster and requires less memory than diagonalizing the full shell-model Hamiltonian, we still need to improve this part in order to perform microscopic level density and reaction calculations simultaneously. Since our calculations do not depend on detailed information on the single-particle structure, a Monte Carlo sampling technique is under development. Instead of calculating the overlap integral for all possible particlehole configurations, we randomly sample the singleparticle states and average them. This could reduce the computational time significantly.

One of the benefits of incorporating the microscopic level density model inside the Hauser-Feshbach code is that we are able to study nuclear reaction in a particular region in a systematic way. The level density depends only on the strength of the effective interaction. When it is estimated from known average spacing values $D_{0}$, we expect that the level densities in that local area can be reasonably predicted. Another benefit is to keep a consistent description of the level densities in the compound and pre-equilibrium processes. Because our level density is constructed as a sum of the partial level densities, $\rho(J)=\rho_{2}(J)+\rho_{4}(J)+\ldots$, the partial level densities can be directly utilized in the pre-equilibrium model calculation. This is particularly important when we revisit the quantum mechanical multistep direct reactions.

In addition to the level density, we envision that many other nuclear reaction model inputs, currently provided 
by an external database or phenomenological parameterization, could be replaced by on-the-fly calculations. For example, when the $\beta$-decay strength function is calculated with the mean-field theory, it gives a population distribution in an initial compound nucleus, from which we can calculate the $\beta$-delayed $\gamma$ and neutron emissions, and fission with Hauser-Feshbach theory [27]. An internal calculation of giant dipole/quadrupole resonances also provides photon strength functions important for the neutron radiative capture process. Combining the level density calculation with the same mean-field model ensures a consistency automatically.

\section{Conclusion}

For better prediction of experimentally unknown nuclear reaction cross sections, we incorporated the mean-field theories into the Hauser-Feshbach code, $\mathrm{CoH}_{3}$. As one of the applications of the mean-field theory, we implemented a microscopic level density model based on the random matrix theory. The calculated level density depends on the effective interaction only, and the calculation is much faster that the shell model calculations. A demonstration was made for ${ }^{208} \mathrm{~Pb}$ using the single-particle states and wave-functions of the FRDM folded Yukawa potential. It was shown that the level densities at low excitation energies are enhanced due to the residual interaction. By studying reasonable and realistic effective interactions, we expect an improvement in the predictive capability of nuclear reaction calculations for nuclei where the experimental information is very scarce, or inexistent.

This work is deeply indebted to S. Yoshida who departed in 2002. The author thanks to H.A. Weidenmüller and P. Talou for their stimulating discussions. This work was carried out under the auspices of the National Nuclear Security Administration of the U.S. Department of Energy at Los Alamos National Laboratory under Contract No. DE-AC52-06NA25396.

\section{References}

[1] R. Capote, M. Herman, P. Obložinský, P.G. Young, S. Goriely, T. Belgya, A.V. Ignatyuk, A.J. Koning, S. Hilaire, V.A. Plujko et al., Nuclear Data Sheets 110, 3107 (2009)

[2] L. Bonneau, T. Kawano, T. Watanabe, S. Chiba, Phys. Rev. C 75, 054618 (2007)

[3] P. Möller, J.R. Nix, W.D. Myer, W. Swiatecki, Atomic Data and Nuclear Data Tables 59, 185 (1995)

[4] T. Kawano, Eur. Phys. J. A 51, 164 (2015)
[5] K. Mizuyama, K. Ogata, Phys. Rev. C 86, 041603 (2012)

[6] G. Blanchon, M. Dupuis, H.F. Arellano, N. Vinh Mau, Phys. Rev. C 91, 014612 (2015)

[7] P. Ring, P. Schuck, "The Nuclear Many-Body Problem," Springer-Verlag Berlin Heidelberg (1980)

[8] H. Feshbach, A. Kerman, S. Koonin, Annals of Physics 125, 429 (1980)

[9] J.L. Ullmann, T. Kawano, T.A. Bredeweg, A. Couture, R.C. Haight, M. Jandel, J.M. O'Donnell, R.S. Rundberg, D.J. Vieira, J.B. Wilhelmy et al., Phys. Rev. C 89, 034603 (2014)

[10] Z. Pluhař, H.A. Weidenmüller, Phys. Rev. C 38, 1046 (1988)

[11] K. Sato, Y. Takahashi, S. Yoshida, Zeitschrift für Physik A Hadrons and Nuclei 339, 129 (1991)

[12] T. Kawano, S. Yoshida, Phys. Rev. C 64, 024603 (2001)

[13] M. Jandel, B. Baramsai, E. Bond, G. Rusev, C. Walker, T.A. Bredeweg, M.B. Chadwick, A. Couture, M.M. Fowler, A. Hayes et al., Eur. Phys. J. A 51, 179 (2015)

[14] N. Fotiades, M. Devlin, R.C. Haight, R.O. Nelson, S. Kunieda, T. Kawano, Phys. Rev. C 91, 064614 (2015)

[15] N. Fotiades, M. Devlin, R.O. Nelson, T. Kawano, J.J. Carroll, Phys. Rev. C 94, 044608 (2016)

[16] T. Tamura, Rev. Mod. Phys. 37, 679 (1965)

[17] P.A. Moldauer, Nuclear Physics A 344, 185 (1980)

[18] T. Kawano, P. Talou, Nuclear Data Sheets 118, 183 (2014)

[19] T. Kawano, P. Talou, H.A. Weidenmüller, Phys. Rev. C 92, 044617 (2015)

[20] T. Kawano, R. Capote, S. Hilaire, P. Chau Huu-Tai, Phys. Rev. C 94, 014612 (2016)

[21] C.A. Engelbrecht, H.A. Weidenmüller, Phys. Rev. C 8, 859 (1973)

[22] T. Kawano, P. Talou, M.B. Chadwick, T. Watanabe, Journal of Nuclear Science and Technology 47, 462 (2010)

[23] L. Bonneau, P. Quentin, D. Samsœn, Eur. Phys. J. A 21, 391 (2004)

[24] M. Bolsterli, E.O. Fiset, J.R. Nix, J.L. Norton, Phys. Rev. C 5, 1050 (1972)

[25] Z. Pluhař, H.A. Weidenmüller, Zeitschrift für Physik A Hadrons and Nuclei 352, 257 (1995)

[26] T. Kawano, S. Chiba, H. Koura, Journal of Nuclear Science and Technology 43, 1 (2006)

[27] T. Kawano, P. Möller, W.B. Wilson, Phys. Rev. C 78, 054601 (2008) 